\title{
"Da minha sala pra sua": teorizando o fenômeno das lives em mídias sociais ${ }^{1}$
}

\author{
Ludmila Lupinacci' \\ https://orcid.org/0000-0002-4371-6768 \\ I - London School of Economics and Political Science - LSE. \\ Londres. Reino Unido.
}

\begin{abstract}
Resumo: Nos últimos anos, as mais populares plataformas de mídias sociais incorporaram a possibilidade de seus usuários criarem, compartilharem e assistirem vídeos ao vivo. Nas chamadas lives, músicos, políticos, influenciadores e pessoas comuns fazem transmissões com variados níveis de improviso e flutuantes esforços de produção, frequentemente a partir do espaço íntimo de seus ambientes domésticos. Este artigo objetiva posicionar criticamente a emergência das lives no contexto da cultura da conectividade, e propor notas teóricas para a compreensão desse fenômeno que ganhou nova visibilidade graças ao isolamento social. São identificados tipos considerados exemplares - aqui denominados lives musicais, conversacionais, instrutivas, de pronunciamento e de companhia. Além disso, são apontados como aspectos centrais às lives o imediatismo, a aparente autenticidade — resultante da relativa imprevisibilidade, mas também de esforços em criar impressões de espontaneidade, familiaridade, intimidade e transparência - e a sensação de experiência compartilhada.
\end{abstract}

Palavras-chave: ao vivo; streaming; conectividade; mídias sociais; plataforma; distanciamento social.

\footnotetext{
Abstract: "From my living room to yours": Theorizing the live streaming phenomenon in social media - Over the last fewyears, the most popular social media platforms have incorporated the possibility for users to create, share, and watch live videos. In so-called 'lives', musicians, politicians, influencers and ordinary people alike broadcast content with varying degrees of improvisation
}

1 Este texto foi colocado originalmente na plataforma preprint do Scielo, com DOl <https://doi. org/10.1590/SciELOPreprints.960> 
and fluctuating production efforts, often from the intimate space of their domestic environments. This article aims to critically position the emergence of this format within the context of the culture of connectivity, and to offer some initial theoretical notes for the understanding of this phenomenon that has acquired new visibility due to the recent need for social distancing. Here, I identify exemplary types of live-streaming videos — which I designate as musical, conversational, instructive, speech, and companionship-based. Then, I characterize as the central features of contemporary live broadcasts their immediacy, apparent authenticity - which in turn results from their relative unpredictability, but also from efforts in producing a sense of spontaneity, intimacy and transparency - and the sense of shared experiencing.

Keywords: live; streaming; connectivity; social media; platform; social distancing.

\section{Introdução}

A principal promessa das tecnologias de comunicação é a de que elas nos ajudariam a ultrapassar as fronteiras do tempo e do espaço, assim acessando o mundo para além de nossas restrições físicas imediatas (CAREY, 1989; SCANNELL 2014). Esse compromisso se torna particularmente evidente quando falamos de mídias eletrônicas, que oferecem ainda mais possibilidades para a concretização de tais fantasias de experiência expandida (MARVIN, 1988). E, de fato, tratando-se de meios como o rádio e a televisão, há uma vasta literatura discutindo tais questões a partir de um atributo específico - o ao vivo.

É importante notar que os termos ao vivo (live) e seu derivado liveness (sem tradução direta para o Português) não foram inicialmente cunhados para fins analíticos ou conceituais (SCANNELL, 2014; VAN ES, 2016), e que, na verdade, seu sentido vem sendo transformado conforme a proliferação de novas tecnologias e seus respectivos usos (COULDRY, 2004; AUSLANDER, 2008). De qualquer modo, quando nos referimos a algum conteúdo ou experiência como ao vivo, normalmente estamos denotando ou a copresença física entre performers e audiência no mesmo tempo e espaço (portanto, "sem intermediação" tecnológica de fato) ou à impressão da não mediação devido a uma transmissão feita em tempo real — isto é, instantaneamente, sem atrasos perceptíveis.

Nos últimos anos, todos os maiores conglomerados do capitalismo digital parecem ter percebido o potencial mercadológico do ao vivo, e passaram a incorporá-lo em seus sites e aplicativos. Do Google/Alphabet, veio 
o YouTube Live; da Amazon, o Twitch; do Facebook, Facebook Live, Instagram, Live Stories e Messenger Rooms; do Twitter, Twitter Live e Periscope; do Snapchat, Live Stories; do Reddit, o RPAN. Isso significa que o termo, até então fortemente associado à transmissão televisiva, passou a fazer parte do portfólio das mais populares plataformas de mídias sociais da contemporaneidade. Ainda assim, até pouco, vídeos ao vivo pareciam secundários frente ao vasto fluxo informacional que compõe o repertório de conteúdo consumido pelo usuário comum - apesar de esforços das plataformas em empurrar live streamings através de suas interfaces e notificações.

Até que chegou março de 2020 e, com ele, o distanciamento social. Apesar do empenho de certas autoridades em deslegitimar o perigo da contaminação massiva pelo COVID-19, boa parte da população passou a ficar em casa por período indeterminado. Ao bagunçar o estado de normalidade e livre circulação de pessoas, a pandemia acaba tornando ainda mais complexas as nossas possibilidades de estar no mundo através das tecnologias de comunicação, trazendo então as experiências ao vivo digitalmente mediadas para o epicentro das nossas rotinas agora primordialmente caseiras. Uma vez em que você se encontra em uma situação de confinamento, qualquer coisa para além da porta de casa vira remota ou distante, e acessível apenas através de tecnologias de comunicação.

A rápida proliferação de lives musicais de artistas dos mais diversos gêneros e orçamentos - além das infames transmissões caseiras minuciosamente despretensiosas de certas figuras públicas - merece esforços de conceituação, e é isso que este trabalho se propõe a fazer. O ponto de partida é uma revisão teórica sintetizada sobre as diferentes atribuições do ao vivo nas áreas dos estudos de performance, televisão e comunicação digital. A partir de tal bibliografia, são identificados seus aspectos centrais em diferentes esferas acadêmicas, e que servirão para guiar o restante da discussão. Em seguida, é apresentada a recente transição discursiva das plataformas de mídias sociais mais populares em direção aos espaços íntimos e privados - exemplificada pelo Facebook e sua busca por deixar de ser o equivalente à praça pública para tornar-se o equivalente à sala de estar (ZUCKERBERG, 2019) - da qual, proponho, a popularização das lives faz parte. Por fim, são identificadas e caracterizadas lives de diferentes tipos performáticos (que classifico como musical, conversacional, instrutiva, de pronunciamento e de companhia), das quais são extraídas as propriedades analíticas mais salientes que, enfim, vão informar a sua tentativa de conceituação enquanto fenômeno midiático e comunicacional. 


\section{Ao vivo, aqui e agora}

Nos estudos de teatro, música e dança, ao vivo implica a copresença física entre quem está performando e sua audiência, e tais publicações tendem a enfatizar a falta de mediação técnica como garantia de um senso de realidade (DIXON, 2007; AGUILAR, 2014). Phelan (1993) toma a impossibilidade de reprodução como a propriedade central da performance ao vivo - a experiência única e efêmera que não pode ser capturada ou revivida. Auslander (2008), por sua vez, aponta que apesar de o ao vivo e o mediado serem normalmente posicionados como opostos, a própria existência do adjetivo ao vivo deriva da emergência das tecnologias de reprodução. Oxímoros como gravado ao vivo evidenciam que liveness é, sobretudo, um termo histórica e tecnologicamente contingente. $\mathrm{O}$ ao vivo seria então mais bem compreendido como uma experiência afetiva dependente de contextos culturais específicos, uma forma particular de fazer parte ou estar envolvido com algum evento ou acontecimento (AUSLANDER, 2008).

Hoje em dia, é mais provável que o entendimento mais difundido do ao vivo esteja atrelado à ideia de transmissão instantânea, em tempo real, graças a seu uso no contexto televisivo. Nesse escopo, Feuer (1983) apresenta um argumento para a compreensão do ao vivo não como a ontologia da TV mas, na verdade, como sua ideologia. Segundo ela, mesmo quando o conteúdo não pode ser considerado live em sentido estrito, a indústria televisiva faz uso do termo com base numa ideologia do espontâneo e do real, que por sua vez estaria ancorada na ideia de que, através da tela, nos é dado acesso direto ao que importa, enquanto tais eventos se desenrolam. Outro a interrogar algumas suposições sobre a televisão é Bourdon (2000), para quem o propósito central da TV seria o oferecer uma experiência compartilhada. Por trás da promessa de conectar pessoas aos eventos do mundo, a indústria estaria criando e reforçando sentimentos de massa e coletividade. Além disso, Bourdon discute a centralidade do contrato mútuo necessário para a experiência ao vivo - para que tal experiência de fato se efetive, é necessário que o espectador subscreva à promessa de um conteúdo enquanto ao vivo. A experiência televisiva e sua relação com o ao vivo são também o tema de interesse de Scannell (2014). Ao analisar tipos diferentes de experiências ao vivo construídas pela indústria televisiva, o autor discute as estratégias de camuflagem dos modos de produção que estão por trás da aparente transparência ou espontaneidade que tendem a caracterizar tais conteúdos. De acordo com ele, o ao vivo é antes de mais nada uma construção, 
que precisa ser trazida à vida através da articulação de capacidade técnica, intencionalidade e sentido. Além disso, Scannell destaca o risco iminente - a (aparente) imprevisibilidade - como parte constituinte do ao vivo em suas diversas configurações.

No contexto das mídias digitais, um dos primeiros acadêmicos a revisitar o conceito de ao vivo foi Couldry (2004), ao propor que sua ideia central seria a de uma potencial conexão a realidades compartilhadas que importam, enquanto elas se desenrolam. Considerando as implicações das tecnologias digitais, ele identifica então novas modalidades de experiências ao vivo, caracterizadas pela possibilidade de copresença descentralizada em relação a um grande núcleo midiático, graças à internet e suas infraestruturas, e ao contato constante possibilitado pela difusão dos dispositivos móveis. Já discutindo o uso do termo no contexto das mídias sociais, Van Es (2016) entende o ao vivo como resultado das ações associadas de instituições, tecnologias e usuários. Para a autora, o ao vivo pode ser observado hoje em diferentes constelações, e teria como eixos centrais o tempo real - compreendido como capacidade técnica, dependente de fatores como velocidade e qualidade da conexão - e o aspecto social. Van Es (2016) salienta ainda que, mesmo nesse contexto capitaneado por plataformas digitais, o ao vivo segue sendo empregado como forma de assegurar os interesses comerciais de grandes empresas.

Dentre as produções que se dedicam ao exame dos live streams contemporâneos, uma significativa parcela é constituída por análises da transmissão de jogos digitais (PAZ; MONTARDO, 2018). Nessa área, destacam-se questões como o trabalho afetivo e performativo empregado por streamers para criar impressões de autenticidade, intimidade e para-socialidade a fim de ampliar sua audiência, engajamento e potencial de monetização (WOODCOCK; JOHNSON, 2019). São também férteis as discussões a respeito do potencial dos vídeos ao vivo para o jornalismo e movimentos populares, em que costumam ser enfatizados o caráter móvel, a estética amadora e o ponto de vista subjetivo de tais conteúdos (KAVADA; TRERÉ, 2019). Por fim, no âmbito da música, a agora difundida transmissão de shows ao vivo é entendida como uma nova possibilidade de fruição ofertada pelas plataformas digitais disponíveis (SÁ; BITTENCOURT, 2014). Em geral, são apontadas como especificidades das performances musicais ao vivo mediadas por plataformas como o YouTube as possibilidades comunicativas à distância, em que ferramentas interativas proporcionam a emergência de micronarrativas 
pessoais dos usuários/espectadores via comentários, reações e tweets, somadas à já familiar estética ao vivo remediada da transmissão televisiva (SÁ; HOLZBACH, 2010).

\section{Da praça pública à sala de estar}

Se as tecnologias midiáticas nos fazem sentir o mundo presente enquanto nos fazem sentir presentes no mundo (FROSH, 2019), parece relevante salientar que, na atual cultura da conectividade (VAN DIJCK, 2013), nossas possibilidades de acesso estão cada vez mais entrelaçadas aos interesses comerciais e à operação de certas plataformas. O termo plataforma em si é controverso, pois tende a ser utilizado pelas companhias do Vale do Silício graças à sua suposta neutralidade (GILLESPIE, 2010). De qualquer forma, por plataforma me refiro aqui às companhias de mídias sociais contemporâneas, que adquiriram enorme alcance e relevância em nossas vidas cotidianas nos últimos anos.

Importante para as ambições teóricas deste trabalho é destacar que, por trás da retórica de conexão, participação e comunidade, tais plataformas têm interesse é no subproduto das nossas constantes interações: nossos dados (VAN DIJCK, 2013). Mídias sociais impactam profundamente em se, como e quando as pessoas têm acesso aos outros e ao mundo, e tendem a apresentar tais processos como naturais, inevitáveis e transparentes, ainda que eles dependam de uma poderosa estrutura computacional (COULDRY; HEPP, 2016). A estratégia de ofuscar a mediação sob o pretexto de oferecer experiências compartilhadas e coletividades naturais não é novidade - porém, ao invés de privilegiar a ideia de massa, mídias sociais tendem a enfatizar o fato de que, através delas, pessoas têm acesso ao que é significativo individualmente (CHUN, 2017; LUPINACCI, 2020).

Parece também interessante notar uma recente transição, em que as mais poderosas plataformas passaram a concentrar suas ambições e recursos em mediar os espaços íntimos e privados de nossas vidas cotidianas (PRIMO et al, 2015). Tal mudança é particularmente curiosa devido ao fato de que, até pouco, a maior delas - o Facebook - vinha escalonando seu discurso de forma a abandonar seu foco original em comunidades locais para abraçar ambições de conexão global. Porém, na nova fase inaugurada nos últimos dois anos, chamada por Mark Zuckerberg (2019) de "a privacy-focused vision for social networking", o interesse está em ocupar espaços particulares. 
O Facebook vem se reposicionando a fim de, nas palavras do próprio CEO, deixar de ser o equivalente à praça pública para tornar-se o equivalente à sala de estar (ZUCKERBERG, 2019). Afinal, segue ele, são espaços reservados que permitem que as pessoas se conectem mais naturalmente. Para alcançar tais objetivos, a companhia traça seis estratégias, dentre as quais estão o foco em interações privadas e a redução da permanência temporal do conteúdo postado, para que usuários sintam-se confortáveis sendo eles mesmos.

Obviamente, trata-se de um exemplo dentre diversas plataformas disponíveis. Porém, devido à posição central que o Facebook - e seus afiliados Instagram, WhatsApp, e Messenger - ocupa no panorama comunicacional contemporâneo, essa mudança tão acentuada merece discussão. O que sugiro é que a adoção de serviços de live streaming por essas plataformas já tão bem-sucedidas - e, antes disso, a incorporação de funcionalidades para a troca de mensagens e narrativas efêmeras (BARROS, 2017) - representa um reposicionamento mais amplo em direção ao conteúdo ordinário e passageiro, e à vida privada, íntima e particular. Cabe lembrar que plataformas não são meros intermediários; são mediadores com agência para moldar os processos comunicacionais que emergem a partir de seus usos (GILLESPIE, 2010; VAN DIJCK, 2013).

É válido ainda considerar que o anunciado reposicionamento da "praça pública" à "sala de estar" traz consigo um duplo deslocamento. Por um lado, ao enfatizar interações efêmeras, íntimas e privadas, plataformas como Facebook passam a privilegiar práticas comunicacionais que, até então, não tinham a centralidade de seu interesse. Nesse sentido, acabam sendo estabelecidas novas possibilidades relacionais que certamente dependem de, mas extrapolam, questões tecnológicas (FERRARA, 2015). Se, conforme apontado na introdução deste trabalho, a ambição central das mídias é o oferecimento de um acesso para além dos limites temporais e espaciais imediatos, novas possibilidades de proximidade e familiaridade acabam sendo criadas. Trata-se, portanto, de uma transformação que toca tanto a transmissão comunicativa quanto o próprio 'comunicar' (BALBI; FERRARA, 2018).

Nesse sentido, a ênfase no conteúdo temporário - que precisa ser consumido aqui e agora - não é acidental. Cabe salientar que, em mídias sociais, efemeridade é quase sempre um atributo transitório: plataformas como Snapchat e Instagram utilizam o termo para encorajar o compartilhamento espontâneo, para que pessoas "sejam elas mesmas" e postem "qualquer 
coisa, a qualquer hora"; meses depois, elas tendem a incorporar ferramentas de arquivamento, ainda que mantenham a aura de descontração, naturalidade e autenticidade. Entendo, portanto, que a recente popularização das lives deve muito, sim, ao isolamento social - mas não apenas a isso. Muito antes da pandemia, Instagram, Twitter e Facebook já vinham priorizando conteúdos ao vivo em suas interfaces e notificações, o que sugere um crescente interesse em interações espontâneas e engajamento quantificável em tempo real.

Ademais, conforme discuti em um trabalho anterior (LUPINACCI, 2019), o ao vivo é descrito pelas plataformas como a melhor solução para conectar pessoas e deixá-las fazer parte daquilo que importa para elas pessoalmente, instantaneamente e independente de distâncias geográficas. Isso significa que as promessas por trás das lives repetem em muito as da mídia tradicional: um compromisso com a experiência efêmera, única e, portanto, autêntica; o acesso imediato ao mundo e seus eventos, enquanto eles acontecem, e como se você estivesse lá, vendo com seus próprios olhos; e a evocação de um senso de experiência compartilhada - o que, em um contexto de isolamento social, torna-se ainda mais atraente.

\section{Assista antes que termine}

"@LidioMateus iniciou uma transmissão ao vivo. Assista antes que termine!" Se o leitor faz uso de mídias sociais, é provável que já tenha se deparado com notificações como essa. Nesta seção, busco identificar e caracterizar de maneira exploratória alguns dos principais tipos de lives circulados hoje. Não tenho a ambição de fornecer uma tipologia exaustiva dos gêneros existentes; a intenção é mapear alguns dos tipos mais populares, a partir dos quais serão extraídas as propriedades mais proeminentes para que, enfim, possamos teorizar o fenômeno de maneira satisfatoriamente embasada.

Ainda que este artigo tenha mais objetivos conceituais do que empíricos, foram adotados alguns procedimentos metodológicos para seu desenvolvimento. Livremente inspirada pela ideia de fluxo midiático (WILLIAMS, 2003 [1972]; SANTOS; PIEDRAS, 2017), acompanhei as recomendações (individualizadas e, portanto, não representativas) de conteúdo ao vivo ofertadas pelo Instagram, Facebook e YouTube durante Abril e Maio de 2020. Baseada em descrições fenomenológicas desenvolvidas por Moores (1995) e Scannell (2014), concentrei meus esforços na observação de como as lives são trazidas 
à vida através de um arranjo de elementos multimodais, em uma tentativa de identificar padrões. Os tipos identificados aqui são: musical, conversacional, instrutivo, de pronunciamento e de companhia.

Live musical é a transmissão ao vivo que tem como foco principal uma performance sonora, realizada por um artista fonográfico ou banda. A relação da indústria da música com o ao vivo é bastante longeva e complexa, já que o termo é utilizado para tratar tanto de apresentações sem intermediação tecnológica como daquelas que são gravadas com um mínimo de pós-produção para manter a estética de crueza (AUSLANDER, 2008). No Brasil, esse tipo de conteúdo parece particularmente popular, e artistas de diversos estilos e públicos vêm oferecendo shows (com variáveis níveis de planejamento e orçamento) para seus fãs. Inclusive, o maior conglomerado de comunicação do país passou a promover diariamente uma lista com as lives do dia, com foco na programação musical.

Já na live conversacional, ainda que os protagonistas possam ser também músicos ou artistas, o foco encontra-se em uma interação dialógica entre dois ou mais participantes. Tais conteúdos por vezes se assemelham a uma entrevista informal, em que um convidado apresenta questões sobre um tema, tópico ou problema específico, e o(s) outro(s) respondem. Em outras ocasiões, o caráter fático é mais preponderante do que a informação ou troca de ideias, e o importante é a manutenção do vínculo comunicativo por si mesmo, mais até do que qualquer que seja o tópico ou assunto discutido (aspecto já apontado como latente em conteúdos efêmeros como as Stories, conforme Barros, 2017).

Com academias fechadas devido à pandemia, houve uma proliferação de vídeo-tutoriais de agachamentos, abdominais e dança das mais variadas modalidades. Os menos afeitos à maromba também não foram abandonados: lições de desenho e maquiagem também se espalharam desde o início do isolamento social. Ainda que tais conteúdos já representassem boa parte do acervo de plataformas como o YouTube e o Instagram (e que, desde pelo menos os anos 80, as vídeoaulas fitness sejam difundidas) a novidade fica por conta da simultaneidade. Na live instrutiva, a intenção é a de simular uma aula presencial, agora mediada por tecnologias digitais. Trata-se, portanto, da tentativa de recriar, ainda que por um curto período, a experiência de uma classe com horário marcado.

Em direção oposta ao viés educacional que frequentemente acompanha as lives instrutivas, nas aqui chamadas lives de pronunciamento a persuasão 
torna-se muito mais preponderante do que o ensino. Nesse gênero, que ganhou nova visibilidade e adesão graças à recusa do Presidente da República em debater e responder formalmente a questões postas pela imprensa, o foco encontra-se no uso de plataformas vistas como alternativas à grande mídia para a disseminação massiva de uma mensagem ou declaração, ainda que por vezes o discurso venha (propositadamente) embalado em uma estética e ambiência informal, caseira e aparentemente improvisada (ainda que minuciosamente planejada).

Por fim, as lives de companhia são aquelas em que, como o nome sugere, o principal objetivo é a manutenção de um laço social ou afetivo com o espectador - em que o 'estar junto' é tão ou mais importante do que o que quer que esteja acontecendo na frente da câmera. Tais conteúdos consistem, basicamente, na transmissão da realização de tarefas domésticas aparentemente banais - comer, cozinhar, ouvir música, meditar, etc. Ainda que vídeos de gameplay (em que o propósito é acompanhar o jogador em seu percurso no jogo) e lifestyle não sejam propriamente uma novidade (eles representam há anos alguns dos mais populares e monetizáveis conteúdos disponíveis em tais plataformas), com o isolamento social obrigatório eles passaram a ganhar nova tração.

Obviamente, há também exemplos que extrapolam tais categorias, e que misturam dois ou mais propósitos em um mesmo conteúdo. Para além das plataformas de mídias sociais mais difundidas, temos ainda o crescimento das watch parties - facilitadas por ferramentas dedicadas como Metastream e Netflix Party, ou da adaptação de outras como o Facebook e Zoom -, em que tanto o caráter conversacional quanto o de companhia (e, dependendo do conteúdo, o musical) são articulados em uma mesma transmissão.

Outro ponto pertinente é o da motivação para a transmissão ao vivo, já que as categorias supracitadas sugerem variados objetivos comunicacionais e mercadológicos. Ainda que seja possível presumir que toda live visa a, de alguma forma, atrair (e segurar) a atenção de espectadores por um determinado período, as finalidades dessas transmissões podem variar do simples prazer em socializar até a tentativa de estabelecer um público específico, seja por motivações políticas (no caso das lives de pronunciamento), seja para construir e engajar uma audiência para o consumo de conteúdo artístico (como é esperado de músicos), ou até mesmo para ampliar o seu alcance dentro das próprias plataformas e, com isso, potencializar o valor 
comercial de seus canais (caso dos influenciadores digitais). Trata-se, portanto, de diferentes articulações daquilo que Baym (2018) designou como trabalho relacional, em que artistas, celebridades e aspirantes fazem uso das plataformas a fim de sustentar interação contínua com seus públicos. Em todo caso, e a despeito de tal heterogeneidade, observo convergências significativas em tais estratégias, o que torna possível uma análise mais detalhada a respeito das dimensões centrais das lives que atravessam tais categorias, como apresentarei em seguida.

\section{Teorizando as lives}

Uma vez mapeados os principais gêneros atualmente circulados, é possível identificar as propriedades mais salientes desse formato em suas variadas configurações. A fim de facilitar a apresentação dos resultados da análise, sintetizo as principais propriedades das lives em quatro categorias: temporalidade, espacialidade, realidade, e sociabilidade.

No que se refere à temporalidade, as lives tendem a enfatizar tanto seu caráter imediato ou instantâneo - tal como o rádio e a televisão (BOURDON, 2000) - quanto aspectos como ineditismo e não reprodutibilidade, típicos das performances não mediadas tecnologicamente (PHELAN, 1993). No entanto, é possível evidenciar as especificidades do novo fenômeno. No que se refere à instantaneidade, as limitações técnicas - como conexão, largura de banda e transmissão de dados - , ainda profusas em dispositivos móveis e mídias sociais, fazem com que os atrasos na comunicação sejam muitas vezes incontornáveis (VAN ES, 2016), e isso é exemplificado pela dificuldade encontrada por duplas sertanejas em realizar suas lives musicais com sucesso. Já a efemeridade, tida como aspecto central da performance ao vivo não tecnologicamente mediada, torna-se uma espécie de persistência programada (BARROS, 2017), uma vez que as plataformas costumam permitir alguma liberdade para que o usuário decida se (e por quanto tempo) o conteúdo inicialmente ao vivo ficará disponível para posterior fruição. Ainda assim, a constante menção ao presente e ao agora, e o fato de que muitas das lives são hoje pré-agendadas para horários específicos, faz com que a sua temporalidade se assemelhe àquela ditada pelo modelo appointmentbased (WILLIAMS, 2003).

Já no que tange a sua espacialidade, as lives atuais tendem, por razões óbvias em um contexto de isolamento social, a ressaltar seu caráter doméstico, tanto na transmissão quanto na recepção. Não quero negar a existência 
de casos em que artistas e influenciadores realizam suas lives em estúdio, com direito a equipe e superprodução. Porém, no contexto da pandemia, nesses casos os responsáveis tendem a ser duramente criticados por "furar a quarentena". Assim, muitas vezes, não há qualquer esforço em fingir que a transmissão ocorre de algum estúdio ou locação externa, e os espaços até então privados da sala de estar e do quarto (ou do jardim, da piscina...) acabam tornando-se palco para concertos, discursos, e talk-shows caseiros. Além disso, o backstage tende a fazer parte da transmissão principal, e fios, cabos, tripés, iluminadores e rebatedores estão visíveis sem tentativa de camuflagem - e a "estética do ao vivo" (SÁ; HOLZBACH, 2010) ajuda a conferir um ar de crueza a tais transmissões. Live streaming em mídias sociais foi, ao menos em sua primeira fase, muito ancorado nas possibilidades de proximidade ofertadas pela mobilidade, e da conexão imediata a partir de uma perspectiva individual (KAVADA; TRERÉ, 2019). Em tempos de distanciamento físico, essa intimidade passa a ser também ancorada na familiaridade dos espaços reservados e domésticos de streamers e espectadores. Já a terceira dimensão identificada como fundamental - o senso de realidade - é construída não pela ausência de mediação técnica, mas pela aparente falta de filtro, edição, pré e pós-producão, que confere a tais conteúdos um ar de rusticidade, espontaneidade e improviso. A estética do-it-yourself é empregada com variados níveis de sutileza, mas mesmo naquelas transmissões em que o orçamento permite um maior esforço de produção, a naturalidade tende a prevalecer devido ao risco e à imprevisibilidade inerentes a um conteúdo midiático não (ou pouco) roteirizado. Autenticidade aqui não depende tanto da camuflagem dos modos de produção a fim de obter um conteúdo em que a mediação pareça inexistente, e sim do ar de vida real que é minuciosamente construído - muitas vezes em contraste com gigantescos anúncios ou product placements de patrocinadores.

Por fim, entendo que o fenômeno das lives contemporâneas tem na sociabilidade seu último eixo fundamental. Por sociabilidade me refiro à sensação de experiência compartilhada; ao senso de coletividade criado e mantido mesmo em um contexto de afastamento físico. É importante notar que, conforme a literatura discutindo o ao vivo no contexto televisivo já indicava, um de seus objetivos principais é o de concentrar, ainda que momentaneamente, a atenção de uma massa de espectadores. A articulação das três primeiras dimensões - temporalidade, espacialidade e realidade - visa, então, criar e promover a ideia de que o que quer que esteja acontecendo 
precisa ser consumido naquele exato momento, e que o próprio fazer parte desse evento com outros que também o fazem é um aspecto central de sua experiência. Além disso, graças às affordances específicas das plataformas de mídias sociais mais difundidas, nas lives a sociabilidade ultrapassa questões para- sociais, e é ancorada nas possibilidades interativas e síncronas ofertadas. Desse modo, especialmente em lives que privilegiam o caráter dialógico (com outro interlocutor ou com o espectador, através de ferramentas como chats em tempo real), a aparente copresença torna-se elemento central.

\section{Considerações finais}

Conforme diz Scannell (2014), estar (ao) vivo hoje está diretamente entrelaçado às possibilidades ofertadas por tecnologias de comunicação. Ainda assim, cabe a ressalva de que, se a experiência tecnologicamente mediada pudesse entregar em termos sensoriais o mesmo que a experiência 'pura', não haveria razão para a incessante busca pelo imediato. Em verdade, o imediatismo - entendido aqui em sua dupla faceta, designando aquilo que é percebido como instantâneo, mas também como o direto, "não mediado" (TOMLINSON, 2007) - é apontado, neste artigo, como elemento central das lives contemporâneas, ao lado da aparente autenticidade (resultante da relativa imprevisibilidade de um conteúdo não editado ou roteirizado, mas também de esforços em criar impressões de espontaneidade, familiaridade, intimidade ou transparência), e da sensação de experiência compartilhada mesmo em ambientes midiáticos que enfatizam a personalização individual.

Historicamente, o ao vivo é utilizado para caracterizar o senso de presença em um contexto remoto; de proximidade em contextos de separação física; de imersão em contextos de domesticidade; de improvisação em contextos de programação; de simultaneidade em contextos de assincronia; de coletividade em contextos de solidão. O ao vivo é, fundamentalmente, a experiência tecnologicamente mediada que, em ao menos alguma de suas dimensões, é percebida como se fosse a experiência direta, imediata, não mediada. O ao vivo é, enfim, o grande como se da comunicação mediada (LUPINACCI, 2020) - o que, em uma situação de isolamento físico forçado, acaba ganhando novo apelo e visibilidade.

A produção acadêmica sobre mídias sociais vem discutindo já há algum tempo como tais plataformas sacrificam aquilo que é considerado privado a fim de expandir e encorajar o compartilhamento público (ainda que a 
nebulosidade da divisão entre tais esferas preceda a difusão dessas tecnologias) (JOHN, 2017; PRIMO et al, 2015). Não pretendo, com isso, sugerir que exista algo essencialmente público ou essencialmente privado - trata-se, na verdade, de reconhecer que, no caso das lives que marcaram especialmente a fase inicial do distanciamento social, a mera exposição de espaços até então socialmente construídos como reservados ao âmbito doméstico faz com que eles passem a pertencer, também, ao que é percebido como ambiente público. A exibição desses ambientes e espaços até então tidos como reservados vai ainda ao encontro do que fora caracterizado como a mercantilização da vida íntima (HOCHSCHILD, 2012; BAYM 2018), em que a intimidade é posta a serviço de objetivos capitalistas, e personalidades, relacionamentos, hobbies e até mesmo quartos, salas e cozinhas são trazidos ao olhar público e convertidos em conteúdo.

Retomando os pressupostos discutidos ao longo do trabalho, parece importante reiterar que, por trás da amplamente difundida retórica de acesso imediato, ilimitado e contínuo, o que de fato move os interesses das plataformas de mídias sociais é o potencial extrativo da conexão prolongada (CHUN, 2017). Um olhar crítico ao encorajamento do compartilhamento e consumo de conteúdo ao vivo mostra, portanto, que tais práticas representam também uma solução para a geração de engajamento quantificável em tempo real. Conforme aponta Van Es (2016), a adoção do ao vivo por companhias como o Facebook e o Google é também uma atualização da historicamente bem-sucedida estratégia que busca assegurar a posição das mídias enquanto mecanismo privilegiado para o acesso imediato ao que de mais relevante acontece no mundo (BOURDON, 2000; COULDRY, 2004).

Tais questões vêm, há pelo menos uma década, sendo examinadas sob a ótica da economia política das mídias (VAN DIJCK 2013). Acredito, no entanto, ser necessário uma sensibilidade centrada na experiência para complementar e complexificar tais debates, a fim de alcançar um olhar a respeito de como de fato essas questões são vividas e percebidas. Nesse sentido, a live contemporânea pode ser entendida como mais do que conteúdo audiovisual ao vivo - é também todo um conjunto de práticas, crenças e percepções em torno de tais materiais. Por fim, se entendemos que relações espaciais são sempre socialmente produzidas, e que mesmo o espaço geográfico possui uma dimensão comunicacional (BALBI; FERRARA, 2018), então a proliferação de lives produzidas e consumidas a partir da sala de estar provoca também a emergência de novos ambientes para a comunicação, cujas especificidades certamente merecem um maior esforço de investigação empírica. 
Ludmila Lupinacci é doutoranda no departamento de Media and Communications da London School of Economics and Political Science (LSE), no Reino Unido.

I.lupinacci-amaral@Ise.ac.uk

\section{Referências}

AGUILAR, A. Negotiating liveness: Technology, economics, and the artwork in LSO live. Music and Letters, 95(2), 2014.

AUSLANDER, P. Liveness. Performance in a mediatized culture. 2nd Edition. Abingdon/ New York: Routledge, 2008.

BALBI, T. M; FERRARA, L. D. Por uma teoria psicogeográfica da comunicação. Intexto, n.41, p.14-34, 2018.

BARROS, L. S. de. Narrativas efêmeras do cotidiano: um estudo das stories no Snapchat e no Instagram. Dissertação de Mestrado. Programa de Pós-Graduação em Comunicação e Informação, Universidade Federal do Rio Grande do Sul, Porto Alegre, 2017.

BAYM, N.K. Playing to the Crowd: musicians, audiences, and the intimate work of connection. New York University Press, 2018.

BOURDON, J. Live television is still alive: on television as an unfulfilled promise. Media, Culture \& Society, 22(5), 2000.

CAREY, J.W. Technology and ideology: The case of the telegraph. Communication as Culture: Essays on Media and Society: Revised Edition, 1989.

CHUN, W.H.K. Updating to remain the same. Habitual New Media. Cambridge, MA: The MIT Press, 2017.

COULDRY, N. Liveness, "Reality," and the Mediated Habitus from Television to the Mobile Phone. The Communication Review, 7(4), 2004.

COULDRY, N.; HEPP, A. The mediated construction of reality. Polity Press, 2016.

DIXON, S. Digital performance: a history of new media in theater, dance, performance art and installation. Cambridge: The MIT Press, 2007.

FERRARA, L. D. Comunicação, mediações, interações. Paulus Editora, 2015.

FEUER, J. The Concept of Live Television: Ontology as Ideology. In: Kaplan E (Ed.), Regarding Television. Los Angeles: American Film Institute, 1983.

FROSH, P. The poetics of digital media. Cambridge: Polity Press, 2019.

GILLESPIE, T. The politics of 'platforms'. New Media \& Society, 12(3), 2010. 
HOCHSCHILD, A.R. The managed heart: Commercialization of Human Feeling. Berkeley: University of California Press, 2012.

JOHN, N.A. The age of sharing. Cambridge: Polity Press, 2017.

KAVADA, A.; TRERÉ, E. Live democracy and its tensions: making sense of livestreaming in the $15 \mathrm{M}$ and Occupy livestreaming in the $15 \mathrm{M}$ and Occupy. Information, Communication \& Society, Online first, 2019.

LUPINACCI, L. 'The closest thing to teleportation': The concept of liveness in the age of connectivity. In: Murru, M.F., Colombo, F., Peja, L., et. al (Eds.) Communication as the intersection of the old and the new. Bremen, Edition Lumière, 2019. Disponível em: <http:// www.researchingcommunication.eu/C05_book14.html>, acesso em 14 de Julho de 2020.

'Absentmindedly scrolling through nothing': liveness and compulsory continuous connectedness in social media. Media, Culture \& Society, 2020. <https://doi. org/10.1177/0163443720939454>

MARVIN, C. When old technologies were new. Thinking about electric communication in the late nineteenth century. New York, Oxford: Oxford University Press, 1988.

MOORES, S. TV discourse and "time-space distanciation": on mediated interaction in modern society. Time \& Society, 4(3), 1995.

PAZ, S.; MONTARDO, S.P. Performance play: consumo digital como performance no streaming the games da plataforma Twitch. Revista Fronteiras, 20(2), 2018.

PHELAN, P. Unmarked: the politics of performance. London: Routledge, 1993.

PRIMO, A.; LUPINACCI, L.; VALIATI, V.; BARROS, L. Comunicação privada na internet: da invenção do particular na Idade Média à hiperexposição na rede. Intexto, 34, 2015.

SÁ, S.P. de; BITTENCOURT, L. Espaços urbanos e plateias virtuais: o YouTube e as transmissões de espetáculos ao vivo. Logos, Edição 40, n. 24, v. 1, 2014.

SÁ, S.P. de; HOLZBACH, A.D. \#u2Youtube e a performance mediada por computador. Revista Galáxia, São Paulo, n. 20, dez. 2010.

SANTOS, N. dos S.; PIEDRAS, E.R. Representações no fluxo televisivo: a naturalização das "novas tecnologias" no jornal, na telenovela e na publicidade. Lumina, 11 (3), 2017.

SCANNELL, P. Television and the meaning of live. Polity Press, 2014.

TOMLINSON, J. The culture of speed: the coming of immediacy. London, Thousand Oaks: Sage, 2007.

VAN DIJCK, J. The culture of connectivity: a critical history of social media. Oxford University Press, 2013.

VAN ES, K. The future of live. Cambridge: Polity Press, 2016. 
WILLIAMS, R. Television. Technology and cultural form. London, New York: Routledge, 2003.

WOODCOCK, J; JOHNSON, M. R. The affective labor and performance of live streaming on Twitch.tv. Television and New Media, 20(8), 2019.

ZUCKERBERG, M. A privacy-focused vision for social networking. Facebook, 2019. Disponível em: <https://www.facebook.com/notes/mark-zuckerberg/a-privacy-focused-vision-for-social-networking/10156700570096634/>. Acesso em: 10 nov. 2020.

Artigo recebido em 02/07/2020 e aprovado em 27/10/2020. 